\title{
The intestinal epithelium is an integral component of a communications network
}

\author{
Martin F. Kagnoff \\ Department of Medicine and Department of Pediatrics, UCSD, La Jolla, California, USA.
}

\begin{abstract}
The epithelial lining of the intestine forms a barrier that separates the intestinal lumen from the host's internal milieu and is critical for fluid and electrolyte secretion and nutrient absorption. In the early 1990s, my laboratory discovered that intestinal epithelial cells could alter their phenotype and produce proinflammatory chemokines and cytokines when stimulated by pathogenic enteric luminal microbes or proinflammatory agonists produced by cells in the underlying mucosa. It is now well accepted that intestinal epithelial cells can be induced to express and secrete specific arrays of cytokines, chemokines, and antimicrobial defense molecules. The coordinated release of molecules by intestinal epithelial cells is crucial for activating intestinal mucosal inflammatory responses as well as mucosal innate and adaptive immune responses. More recent studies have focused on the intestinal epithelial signaling pathways that culminate in immune activation as well as the role of these pathways in host defense, mucosal injury, mucosal wound healing, and tumorigenesis. The emerging picture indicates that intestinal epithelial cells represent an integral component of a highly regulated communications network that can transmit essential signals to cells in the underlying intestinal mucosa, and that intestinal epithelial cells, in turn, serve as targets of mucosal mediators. These signals are essential for maintaining intestinal mucosal defense and homeostasis.
\end{abstract}

\section{The intestinal epithelium: more than a physical barrier}

Prior to studies from our laboratory in the early 1990s, the intestinal epithelium that separates the intestinal lumen from the underlying intestinal mucosa was viewed as a physically protective barrier, consisting of a single layer of epithelial cells that functioned to regulate the absorption of nutrients and fluid as well as electrolyte absorption and secretion. Moreover, the intestinal mucosa underlying this single-cell-thick epithelial cell barrier was known to contain abundant cell types of the innate and adaptive immune systems, such as macrophages, DCs, T cells, and B cells, that could participate in mucosal inflammation and immunity.

Breakthrough studies in my laboratory, including our 1995 report in the JCI, led to the discovery that in addition to separating the host's external luminal environment from the host's internal mucosal milieu, the intestinal epithelial cells could, under varying conditions, alter their phenotype

Conflict of interest: The author has declared that no conflict of interest exists.

Citation for this article: J Clin Invest. 2014; 124(7):2841-2843. doi:10.1172/JCI75225. and produce proinflammatory mediators (1-3). These initial studies revealed that infection of intestinal epithelial cells with enteroinvasive enteric microbial pathogens, or stimulation of epithelial cells with proinflammatory mediators generated by cells in the underlying mucosa, resulted in the rapid upregulation of gene expression and ensuing production of proinflammatory cytokines and chemokines by intestinal epithelial cells (1-4). At the time, the concept that epithelial cells could activate inflammatory and immune responses against microbial pathogens was considered counterintuitive and met with surprise and doubt. After all, weren't intestinal epithelial cells supposed to play a role in barrier function and mucosal protection from inflammation, not initiate mucosal inflammation? In a series of subsequent publications, we demonstrated that intestinal epithelial cells not only produce a broad array of cytokines and chemokines (Figure 1A), but they also express receptors for cytokines and chemokines and produce antimicrobial peptides. The epithelial cells, which form a border separating the intestinal lumen from the underlying mucosa, represent an integral component of an elaborate communications system that transmits signals between the host's external environment and the underlying cells of the host's innate and adaptive mucosal immune system. Subsequently, the intestinal epithelial cells respond to the signals generated by cells of the intestinal mucosa (Figure 1B and refs. 4-14).

\section{Intestinal epithelium-mediated mucosal defense and homeostasis}

The implications and the impact of our 1995 JCI article, "A distinct array of proinflammatory cytokines is expressed in human colon epithelial cells in response to bacterial invasion" (3), were profound, in that this and subsequent studies demonstrated that intestinal epithelial cells were important for the initiation and regulation of mucosal inflammation and host defense against enteric microbial pathogens with the ability to invade, inject microbial products into, or activate surface receptors on epithelial cells (15, $16)$. We now know that if early epithelialdependent cell signals are not produced, enteric pathogens generate less mucosal inflammation; however, reduced inflammation can concurrently result in greater systemic spread of infection and may lead to greater morbidity and death (17).

The times leading up to and following the publication of our $1995 \mathrm{JCI}$ article were exciting. Each new discovery by my laboratory and others led to important new functional insights into the role of the intestinal epithelium in mucosal defense and homeostasis. It was not long until we and others began to question what mechanisms turn off the proinflammatory phenotype of the intestinal epithelium and to investigate the nature of interactions between the epithelium and noninvasive epithelial adherent enteric microbes, parasites that associate with or invade intestinal epithelium, and lumen-dwelling commensal microbes, the latter having been largely ignored before. Concepts derived from these studies further led to years of work in our laboratory and others interested in the molecular complexity of intestinal epithelial cell signaling and functions, the balance between 
A
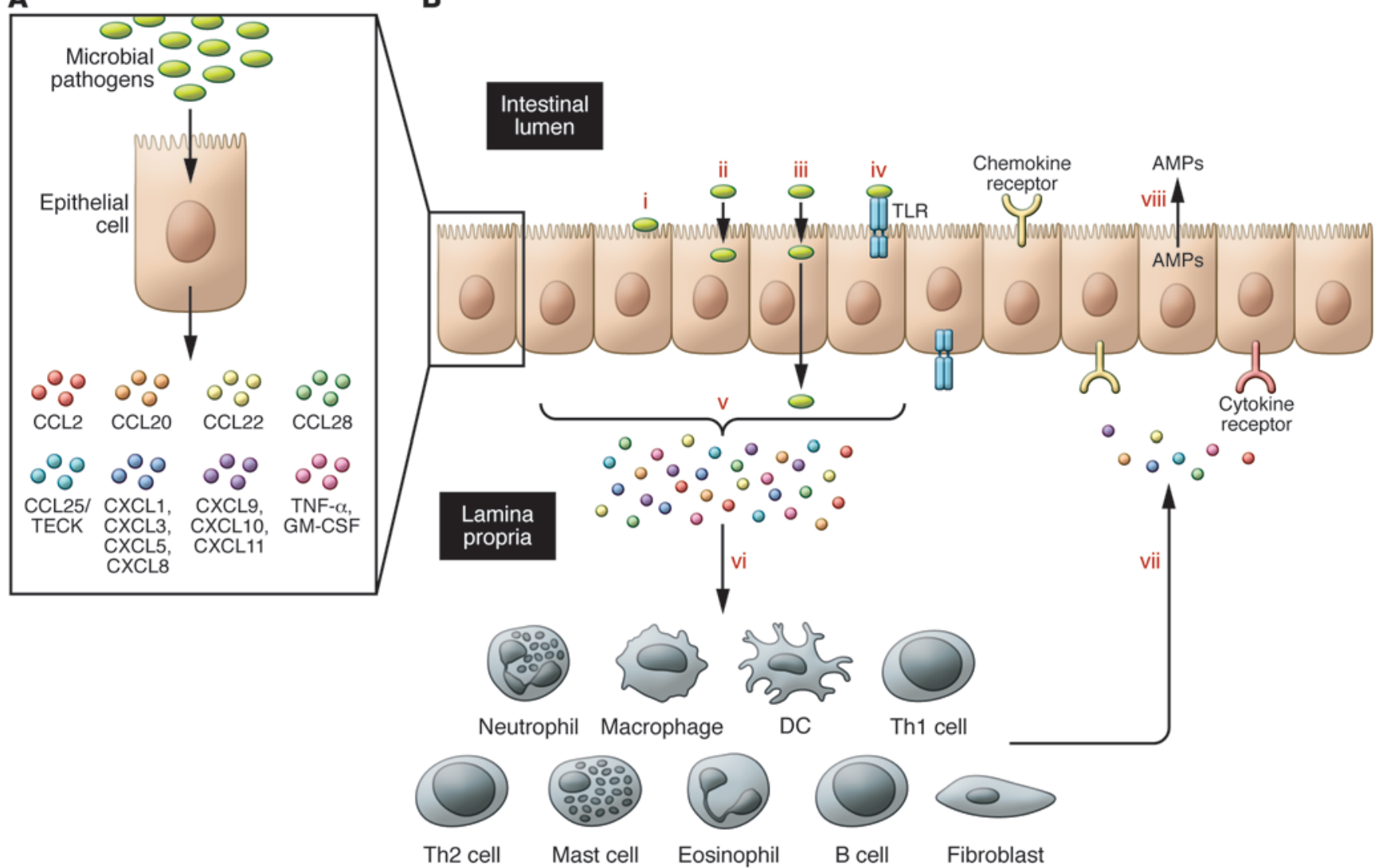

Figure 1

Epithelial cells represent an integral component of a communications network. (A) Intestinal epithelial cells can be induced to express chemokines and cytokines in response to encounter with enteric microbial pathogens. These include chemokines that chemoattract neutrophils (CXCL8, CXCL1, CXCL3, and CXCL5; dark blue), macrophages and DCs (CCL2; red), DCs and memory T cells (CCL20; orange), DCs and Th2 cells (CCL22; yellow), Th1 cells (CXCL9, CXCL10, and CXCL11; purple), plasma cells (CCL28; green), $\alpha 4 \beta 7$ T cells (CCL25, also known as TECK; light blue), and cytokines (e.g., TNF- $\alpha$ and GM-CSF; magenta). (B) Enteric microbial pathogens in the intestinal lumen can associate with the epithelial cell surface (i), invade epithelial cells and reside within those cells (ii), invade epithelial cells and the underlying mucosa (lamina propria) (iii), or activate surface receptors, such as TLRs (iv). In response, the intestinal epithelium can change its phenotype to produce chemokines and cytokines (v) that act on underlying cells of the innate and adaptive immune system in the lamina propria (vi). Cells in the lamina propria, in turn, produce mediators (vii) that act on cytokine and chemokine receptors on intestinal epithelial cells. Epithelial cells also express TLRs that respond to microbial products (e.g., bacterial flagellin signals through TLR5) and chemokine receptors (CCR6, CXCR4, CCR5, and CX3CR1) and can be induced to produce antimicrobial peptides (AMPs), such as -defensins and cathelicidin (viii).

pro- and antiinflammatory signals, the yin and yang of microbial-epithelial interactions in terms of benefit and liability to the host and pathogen, and how enteroinvasive pathogens that use different receptors and mechanisms for host epithelial cell entry and have different "intracellular lifestyles" use common signaling pathways (e.g., NF-кB, MAPKs) to activate mucosal mediators $(18,19)$. We now widely appreciate that the intestinal epithelium is an active participant, acting as "communication central," in the interactions among enteric pathogens, the commensal microbiome, and the host's internal milieu. More recent studies of microbial commensals has led to greater understanding of the human microbiome and its role in human physiology and metabolism and sparked a major national effort to study and determine the function of the gut bacteria and their role in human health and disease. Furthermore, other studies have shed light on the role of epithelial signaling and cytokines on epithelial protection from injury $(20,21)$, wound healing (22), and tumorigenesis (23).

\section{Concluding remarks}

While much has been learned about the interactions between microbes and the intestinal epithelium, complete therapeutic translation remains elusive. Major challenges remain before this knowledge can be used to develop effective targeted therapeutic approaches to prevent, modulate, or cure enteric infections, ameliorate inflammatory diseases of the intestinal tract and extraintestinal regions, and relieve autoimmune diseases whose pathogenesis originates from interactions involving intestinal luminal events and the intestinal epithelium.

\section{Acknowledgments}

I acknowledge the contribution of the many dedicated postdoctoral fellows I have had the privilege to train in my laboratory over the past years, the postdoctoral fellows I have worked with from Michael Karin's laboratory, and especially Lars Eckmann, with whom I have had a 
long-term collaboration dating back to the days he was a postdoctoral fellow in my laboratory. These studies would not have been possible without continuous support from the NIDDK, NIH.

Address correspondence to: Martin F. Kagnoff, University of California at San Diego, 9500 Gilman Drive, La Jolla California 92093, USA. Phone: 858.534.4622; Fax: 858.534.5691; E-mail: mkagnoff@ucsd.edu.

1. Eckmann L, Jung HC, Schürer-Maly C, Panja A, Morzycka-Wroblewska E, Kagnoff MF. Differential cytokine expression by human intestinal epithelial cell lines:regulated expression of interleukin 8 . Gastroenterology. 1993; 105(6):1689-1697.

2. Eckmann L, Kagnoff MF, Fierer J. Epithelial cells secrete the chemokine interleukin-8 in response to bacterial entry. Infect Immun. 1993;61(11):4569-4574.

3. Jung HC, et al. A distinct array of proinflammatory cytokines is expressed in human colon epithelial cells in response to bacterial invasion. J Clin Invest. 1995;95(1):55-65.

4. Yang SK, Eckmann L, Panja A, Kagnoff MF. Differential and regulated expressionof C-X-C, C-C, and $\mathrm{C}$-chemokines by human colon epithelial cells. Gastroenterology. 1997;113(4):1214-1223.

5. Berin MC, Dwinell MB, Eckmann L, Kagnoff MF. Production of MDC/CCL22 by human intestinal epithelial cells. Am J Physiol Gastrointest Liver Physiol. 2001;280(6):G1217-G1226.

6. Izadpanah A, Dwinell MB, Eckmann L, Varki NM, Kagnoff MF. Regulated MIP-3 /CCL20 production by human intestinal epithelium: mechanism for modulating mucosal immunity. Am J Physiol
Gastrointest Liver Physiol. 2001;280(4):G710-G719.

7. Dwinell MB, Lügering N, Eckmann L, Kagnoff MF. Regulated production of interferon-inducible T-cell chemoattractants by human intestinal epithelial cells. Gastroenterology. 2001;120(1):49-59.

8. Eckmann L, Smith JR, Housley MP, Dwinell MB, Kagnoff MF. Analysis by high density cDNA arrays of altered gene expression in human intestinal epithelial cells in response to infection with the invasive enteric bacteria Salmonella. J Biol Chem. 2000;275(19):14084-14094.

9. Ogawa H, Iimura M, Eckmann L, Kagnoff MF Regulated production of the chemokine CCL28 in human colon epithelium. Am J Physiol Gastrointest Liver Physiol. 2004;287(5):G1062-G1069.

10. Kagnoff MF, Eckmann L. Epithelial cells as sensors for microbial infection. J Clin Invest. 1997;100(1):6-10.

11. Dwinnel MB, Eckmann L, Leopard JD, Varki NM, Kagnoff MF. Chemokine receptor expression by human intestinal epithelial cells. Gastroenterology. 1999;117(2):359-367.

12. O'Neil DA, et al. Expression and regulation of the human beta-defensins hBD-1 and hBD-2 in intestinal epithelium. I Immunol. 1999; 163(12):6718-6724.

13. Hase K, Eckmann L, Leopard JD, Varki N, Kagnoff MF. Cell differentiation is a key determinant of cathelicidin LL-37/human cationic antimicrobial protein 18 expression by human colon epithelium. Infect Immun. 2002;70(2):953-963.

14. Iimura M, Gallo RL, Hase K, Miyamoto Y, Eckmann L, Kagnoff MF. Cathelicidin mediates innate intestinal defense against colonization with epithelial adherent bacterial pathogens. J Immunol. 2005;174(8):4901-4907.

15. Berin MC, Darfeuille-Michaud A, Egan LJ, Miyamoto Y, Kagnoff MF. Role of EHEC O157:H7 virulence factors in the activation of intestinal epithelial cell NF-KB and MAP kinase pathways and the upregulated expression of interleukin 8 . Cell Microbiol. 2002;4(10):635-648.

16. Miyamoto Y, Iimura M, Kaper JB, Torres AG, Kagnoff MF. Role of Shiga toxin versus $\mathrm{H} 7$ flagellin in enterohaemorrhagic Escherichia coli signalling of human colon epithelium in vivo. Cell Microbiol. 2006;8(5):869-879.

17. Sansonetti PJ, Arondel J, Huerre M, Harada A, Matsushima K. Interleukin-8 controls bacterial transepithelial translocation at the cost of epithelial destruction in experimental shigellosis. Infect Immun. 1999;67(3):1471-1480.

18. Elewaut D, DiDonato JA, Kim JM, Truong F, Eckmann L, Kagnoff MF. NFKB is a central regulator of the intestinal epithelial cell innate immune response induced by infection with enteroinvasive bacteria. J Immunol. 1999;163(3):1457-1466.

19. Guma M, et al. Constitutive intestinal NF-кB does not trigger destructive inflammation unless accompanied by MAPK activation. J Exp Med. 2011; 208(9):1889-1900.

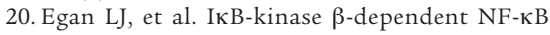
activation provides radioprotection to the intestinal epithelium. Proc Natl Acad Sci U S A. 2004; 101(8):2452-2457.

21. Egea L, McAllister CS, Lakhdari O, Minev I, Shenouda S, Kagnoff MF. GM-CSF produced by nonhematopoietic cells is required for early epithelial cell proliferation and repair of injured colonic mucosa. J Immunol. 2013;190(4):1702-1713.

22. Egan LJ, de Lecea A, Lehrman ED, Myhre GM, Eckmann L, Kagnoff MF. Nuclear factor- $\kappa$ B activation promotes restitution of wounded intestinal epithelial monolayers. Am J Physiol Cell Physiol. 2003;285(5):C1028-C1035.

23. Greten FR, et al. IKK $\beta$ links inflammation and tumorigenesis in a mouse model of colitis-associated cancer. Cell. 2004;118(3):285-296. 